\title{
Algorithms for graphs embeddable with few crossings per edge
}

Citation for published version (APA):

Grigoriev, A., \& Bodlaender, H. L. (2004). Algorithms for graphs embeddable with few crossings per edge. (UU-CS-2004-058 ed.) University Utrecht. Computing Science Technical Report No. 058

Document status and date:

Published: 01/01/2004

Document Version:

Publisher's PDF, also known as Version of record

\section{Please check the document version of this publication:}

- A submitted manuscript is the version of the article upon submission and before peer-review. There can be important differences between the submitted version and the official published version of record.

People interested in the research are advised to contact the author for the final version of the publication, or visit the DOI to the publisher's website.

- The final author version and the galley proof are versions of the publication after peer review.

- The final published version features the final layout of the paper including the volume, issue and page numbers.

Link to publication

\footnotetext{
General rights rights.

- You may freely distribute the URL identifying the publication in the public portal. please follow below link for the End User Agreement:

www.umlib.nl/taverne-license

Take down policy

If you believe that this document breaches copyright please contact us at:

repository@maastrichtuniversity.nl

providing details and we will investigate your claim.
}

Copyright and moral rights for the publications made accessible in the public portal are retained by the authors and/or other copyright owners and it is a condition of accessing publications that users recognise and abide by the legal requirements associated with these

- Users may download and print one copy of any publication from the public portal for the purpose of private study or research.

- You may not further distribute the material or use it for any profit-making activity or commercial gain

If the publication is distributed under the terms of Article $25 \mathrm{fa}$ of the Dutch Copyright Act, indicated by the "Taverne" license above, 


\title{
Algorithms for graphs embeddable with few crossings per edge
}

\author{
Alexander Grigoriev \\ Hans L. Bodlaender
}

institute of information and computing sciences, utrecht university

technical report UU-CS-2004-058

www.cs.uu.nl 


\title{
Algorithms for graphs embeddable with few crossings per edge*
}

\author{
Alexander Grigoriev ${ }^{\dagger} \quad$ Hans L. Bodlaender ${ }^{\ddagger}$
}

\begin{abstract}
We consider graphs that can be embedded on a surface of bounded genus such that each edge has a bounded number of crossings. We prove that many optimization problems, including maximum independent set, minimum vertex cover, minimum dominating set and many others, admit polynomial time approximation schemes when restricted to such graphs. This extends previous results by Baker [1] and Eppstein [7] to a much broader class of graphs. We also show that testing if a graph can be drawn in the plane with at most one crossing per edge is NP-complete.
\end{abstract}

\section{Introduction}

Already more than two decades ago, Baker [1] showed that the maximum independent set and many other NP-hard optimization problems on graphs admit polynomial time approximation schemes (PTAS) when restricted to planar graphs. The basic idea of Baker's algorithm was to remove the vertices in every $k$ th level of a breadth first search tree (BFS) and to solve the problem on the remaining components by a dynamic programming algorithm. Baker proved that from $k$ ways of choosing which set of levels to remove there is at least one which only decreases the size of the maximum independent set by a factor of at most $(k-1) / k$. Moreover, remaining components after levels deletion are $k$-outerplanar graphs, and dynamic programming can solve the problem on these components efficiently.

Recently, Eppstein in [7] observed that the results by Baker [1] can be extended to any minor-closed family of graphs satisfying so-called diameter-treewidth property. This implies that the problem admits a PTAS if restricted to bounded-genus graphs. This result

*This research has been carried out as part of the project "Tree Width and Combinatorial Optimization (TACO)" supported by the Netherlands Organization for Scientific Research NWO.

${ }^{\dagger}$ Maastricht University, Faculty of Economics and Business Administration, Quantitative Economics, P.O. Box 616, 6200 MD Maastricht, The Netherlands, Email: a.grigoriev@ke.unimaas.nl

${ }^{\ddagger}$ Institute of Information and Computing Sciences, Utrecht University, Padualaan 14, De Uithof, P.O. Box 80089, 3508 TB Utrecht, The Netherlands, Email: hansb@cs.uu.nl 
has been generalized to other minor-closed classes; in particular, Grohe gave PTAS's for several problems, for any minor-closed family that does not contain all graphs [6].

Nowadays, there is a growing body of work, mainly developed by Demaine and Hajiaghayi, based on the concept of "bidimensionality" and presenting directions for generalizations of the Baker-Eppstein ideas of using a diameter like parameter to bound treewidth and thus yielding polynomial time approximation schemes for problems on even more general classes of graphs; see Demaine et al. in [2, 3, 4].

In this paper we continue the line of investigations - in which way can Baker's technique be further extended? Revisiting Eppstein [7] result, we observe that the restriction that the class of graphs must be minor-closed can be relaxed. By moving from the input graph to an auxiliary graph obtained by replacing each crossing by a vertex and back, we can obtain Baker-type PTAS's for several problems on graphs that are embeddable on a surface of bounded genus (e.g., the plane, the torus) with a bounded number of crossings per edge. We emphasize on the fact that all known results, also in Demaine et al. [2, 3, 4], work only under assumption that the graph family is minor-closed. In contrast, in this paper, we introduce the graph families on which Baker-Eppstein techniques work perfectly but actually any graph is a minor of sufficiently large graph of the considering families.

In the end of the paper we present several additional results which provide an insight on the graphs with few crossings per edge.

\section{Problem and Definitions}

We illustrate the basic ideas of the PTAS on the maximum independent set problem. Given a graph $G=(V, E)$, we look for a maximum cardinality independent set in $G$, i.e., a vertex subset $V^{\prime} \subseteq V$ such that no two vertices from $V^{\prime}$ are adjacent by an edge from $E$. This problem is known to be NP-hard even for planar graphs. The problem admits a PTAS if restricted to planar graphs [1] and even to bounded-genus graphs [7]. Let $n=|V|$.

Definition 2.1 (Good embedding). We call an embedding of graph $G$ on a surface $S$ of genus $g$ a good embedding if it satisfies the following conditions: (i) all vertices of the graph are given as distinct points in $S$; (ii) no two edge crossings happen in the same point in S; (iii) for any edge no vertex of the graph, except the endpoints of the edge, is situated on the edge.

Definition 2.2 (Crossing parameter). Let the crossing parameter $\varphi$ of a graph (on surface $S$ ) be the minimum over all good embeddings on $S$ of the maximum over all edges $e$ of the number of edge crossings of $e$.

Through this paper we assume that a good embedding of $G$ is given and both the crossing parameter $\varphi$ and the genus $g$ of $S$ are bounded by some constants. Clearly, the graph is planar if $g=0$ and $\varphi=0$. 
Definition 2.3 (Tree decomposition). $A$ tree decomposition $\left(\left\{X_{i} \mid i \in I\right\}, T=(I, F)\right)$ of a graph $G=(V, E)$ is a pair, with $\left\{X_{i} \mid i \in I\right\}$ a collection of subsets of $V$ (called bags), such that

- $\bigcup_{i \in I} X_{i}=V$.

- For all $\{v, w\} \in E$, there is an $i \in I$ with $v, w \in X_{i}$.

- For each $v \in V$, the set $T_{v}=\left\{i \in I \mid v \in X_{i}\right\}$ forms a connected subtree of $T$.

Definition 2.4 (Treewidth). The width of a tree decomposition $\left\{X_{i} \mid i \in I\right\}$ is $\max _{i \in I}\left|X_{i}\right|-$ 1. The treewidth of a graph $G$ is the minimum width over all tree decompositions of $G$.

\section{The Polynomial Time Approximation Scheme}

We now describe our polynomial time approximation scheme for the maximum independent set problem on graphs with bounded crossing parameter on bounded genus. We assume the embedding is given. Consider the following algorithm $\mathcal{A}$ which is a revised version of the algorithms by Baker [1] and Eppstein [7].

Input: Graph $G$, parameter $k$ (without loss of generality, let $\varphi<k$ ).

\section{Algorithm $\mathcal{A}$ :}

1. Construct the graph $G^{\prime}=\left(V^{\prime}, E^{\prime}\right)$ obtained from $G$ by replacing each edge crossing by a vertex.

2. Build a breadth first search tree $T$ of $G^{\prime}$, with an arbitrary root $v_{0}$, and consider the levels of the tree (i.e., vertex sets with equal distance to $v_{0}$ ).

3. For all $i, 0 \leq i \leq k$, we perform the following procedure.

(a) Remove from $G^{\prime}$ all levels of $T$ congruent to $i(\bmod k)$ together with their $\varphi$ successive levels. This decomposes $G^{\prime}$ into a collection of subgraphs $\mathcal{H}=$ $\left\{H_{1}, H_{2}, \ldots, H_{r}\right\}$ where each subgraph $H_{t}=\left(V_{t}, E_{t}\right)$ is induced by $k-\varphi-1$ consecutive levels in $T$ of $G^{\prime}$.

(b) Consider a subgraph $G_{t}$ of $G$ induced by vertices $V_{t} \cap V$. Since the number of crossings per edge is at most $\varphi$ and we removed $\varphi+1$ consecutive levels from $G^{\prime}$, we have that after deletion of levels there is no an edge $e \in E$ such that its two endpoints belong to two different subgraphs $G_{t^{\prime}}$ and $G_{t^{\prime \prime}}$. Therefore, for each $i, 0 \leq i \leq k$, we have a subgraph of $G$ formed by a collection of disconnected subgraphs $G_{1}, G_{2}, \ldots, G_{r}$. By Lemma 3.1 below, the treewidth of $G_{t}$ is bounded by $O(k)$ for all $t=1,2, \ldots, r$. Hence, the maximum independent set for $G_{t}$ can be found in time $O\left(n 2^{O(k)}\right)$ by a dynamic programming algorithm, using standard treewidth techniques; see, e.g., Telle and Proskurowski [12]. 
(c) Let $S_{i}$ be a union of the maximum independent sets of all $G_{t}, t=1,2, \ldots, r$.

4. Define $S_{\max }$ by a maximum cardinality set over all $S_{i}, 0 \leq i \leq k$.

\section{Output: Return $S_{\max }$.}

The following lemma is a key for algorithm $\mathcal{A}$ and for the main result of the paper.

Lemma 3.1. The treewidth of $G_{t}$ is bounded by $O(k)$ for all $t=1, \ldots, r$.

Proof. Consider a subgraph $H_{t}$ induced by levels $r+1, r+2, \ldots, r+s$ in $T$ of $G^{\prime}$ where $s=k-\varphi-1=O(k)$. Consider a minor of $G^{\prime}$ obtained by contraction of the first $r$ levels in $T$ to a single vertex and deletion of all levels above $r+s$. Clearly, this minor is a graph of genus $g$. Moreover, it has a diameter of at most $2(k-\varphi-1)=O(k)$. By Eppstein [7] the treewidth of such a minor is $O(g k)$. Therefore, $H_{t}$ as a subgraph of such a minor has the treewidth of at most $O(g k)$ as well.

Now, let us estimate how much the treewidth of $G_{t}$ and $H_{t}$ can differ. Construct a graph $H_{t}^{\prime}$ from $H_{t}$ by replacing each vertex $v$ in $H_{t}$ that represents an edge crossing, say $e_{1}$ and $e_{2}$, by two adjacent vertices $v_{1}$ and $v_{2}$ representing $e_{1}$ and $e_{2}$ respectively. Let $v_{1}$ be adjacent to all vertices corresponding to the neighborhood of $v$ representing $e_{1}$, and let $v_{2}$ be adjacent to all vertices corresponding to the neighborhood of $v$ representing $e_{2}$. A tree decomposition of $H_{t}$ of treewidth $d$ can be turned into a tree decomposition of $H_{t}^{\prime}$ of treewidth at most $2 d+1$, by replacing each occurrence of an vertex that represents a crossing of two edges in a bag by the corresponding two vertices; this gives a tree decomposition of $H_{t}^{\prime}$ whose maximum bag size is at most doubled. One can also observe that we can select for each edge in $G_{t}$ a path in $H_{t}^{\prime}$ between its endpoints, such that these paths do not have internal vertices in common. Thus, $G_{t}$ is a minor of $H_{t}^{\prime}$ and hence the treewidth of $G_{t}$ is at most twice the treewidth of $H_{t}$ plus one, and thus $O(g k)=O(k)$ as required.

Now, we are ready to summarize the main results of the paper in the following theorem and corollary.

Theorem 3.1. Algorithm $\mathcal{A}$ outputs an independent set of graph $G$ of size at least 1 $O(1 / k)$ times the optimum in time $O\left(k n 2^{O(k)}\right)$, and thus, there is a PTAS for maximum independent set for graphs given with an embedding on a surface of bounded genus and with bounded crossing parameter.

Proof. Since for all $i, 0 \leq i \leq k$, set $S_{i}$ is a union of independent sets of disconnected subgraphs of $G$, Algorithm $\mathcal{A}$ returns an independent set of graph $G$.

As in Baker [1], there is at least one $i, 0 \leq i \leq k$, such that at most $(\varphi+1) / k$ of the nodes in the optimal solution are at the level that is congruent to $i(\bmod k)$. This implies that $\left|S_{\max }\right|$ is approximating the optimum within a factor $(k-\varphi-1) / k=1-O(1 / k)$.

Notice that the most time consuming operation in Algorithm $\mathcal{A}$ is the dynamic programming used in step 3. As we already noticed above, this dynamic programming requires 
$O\left(n 2^{O(k)}\right)$ time. Since we run step 3 for all choices of $i, 0 \leq i \leq k$, the total running time of Algorithm $\mathcal{A}$ is $O\left(k n 2^{O(k)}\right)$.

Corollary 3.1.1. For each of the following problems (and many others) there is a PTAS for graphs embeddable on a surface of bounded genus with bounded crossing parameter:

- minimum vertex cover;

- minimum dominating set;

- minimum edge dominating set;

- minimum triangle matching;

- maximum H-matching;

- maximum tile salvage.

Proof. This can be proven in the same way as Theorem 3.1, using techniques similar to those of Baker [1].

\section{More on the Crossing Parameter}

Graphs with bounded crossing parameter were investigated by several authors in the context of graph drawing; see, e.g., Pach and Toth [11]. However, before this article nothing was known on the recognition complexity of the graphs with small crossing parameter. To give the reader more insight on the graphs with few crossings per edge, in this section we present some results on the computational complexity of the crossing parameter and some other useful properties of the class of graphs with bounded crossing parameter.

Theorem 4.1. The problem to determine if a given graph $G$ can be embedded on the plane with crossing parameter 1 is NP-complete.

Proof. We prove the theorem by reduction of the well known strongly NP-complete problem 3-PARTITION; see Garey and Johnson [5]: Given a set $A$ of $3 m$ elements, a bound $B \in$ $\mathbb{Z}^{+}$, and a size $s(a) \in \mathbb{Z}^{+}$for each $a \in A$ such that $B / 4<s(a)<B / 2$ and such that $\sum_{a \in A} s(a)=m B$, can $A$ be partitioned into $m$ disjoint sets $A_{1}, A_{2}, \ldots, A_{m}$ such that for $1 \leq i \leq m, \sum_{a \in A_{i}} s(a)=B ?$

Before starting the reduction, we would like to mention several properties of the complete graph on six vertices: (i) by Guy's conjecture proven for complete graphs on up to 10 vertices, see, e.g., [13], $K_{6}$ has crossing number 3 ; (ii) $K_{6}$ can be drawn in the plane with three crossings, at most one crossing per edge, and two vertices in the exterior, see Figure 1; (iii) in any drawing of $K_{6}$ having at most one crossing per edge for any two vertices of the graph there is a path between those two vertices such that all edges of the path are 
crossed. For a proof of (iii) see Theorem 6.1 in Appendix. Taking into account properties (i)-(iii), we can use graph $K_{6}$ as an edge that cannot be crossed. In the figures below thick edges are graphs $K_{6}$.
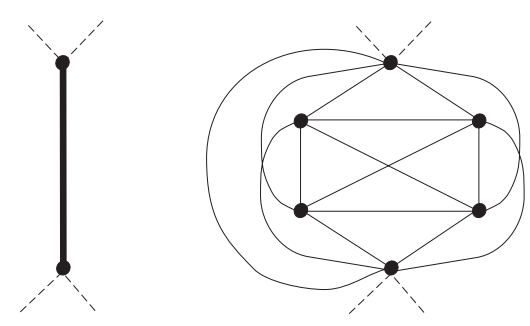

Figure 1: Thick edge is a graph $K_{6}$.

Now, we reduce 3-PARTITION to the crossing parameter 1 recognition. Given an instance of 3-PARTITION, we construct the graph for the crossing parameter 1 recognition as follows. For each element $a \in A$ we introduce a gadget $P_{a}$ called splitter which is a simple star having $s(a)+1$ edges. We also introduce two special gadgets called transmitter and collector. Both these gadgets have a "double"-wheel form with $3 m$ thick radials for the transmitter and $B m$ thick radials for the collector; see Figure 2 .
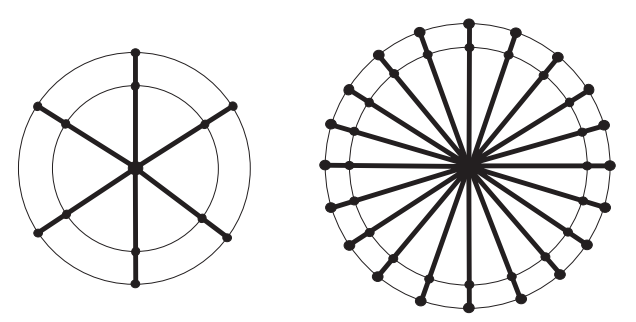

Figure 2: $m=2$ transmitter and $B=10, m=2$ collector.

We finish construction by adding the following edges:

- We connect the transmitter center to a degree 1 vertex of each splitter $P_{a}, a \in A$.

- We connect remaining $s(a)$ degree 1 vertices of each splitter $P_{a}, a \in A$, to the collector center.

- Let a cycle $\left[t_{1}, t_{2}, \ldots, t_{3 m}\right]$ be an exterior circuit of the transmitter and a cycle $\left[c_{1}, c_{2}, \ldots, c_{B m}\right]$ be an exterior circuit of the collector. For all $i \in\{1,2, \ldots, m\}$, connect vertex $t_{3 i}$ to vertex $c_{B i}$ by a thick edge; for illustration see Figure 3.

Let us refer to the obtained graph as to $G$. Now, we claim that $G$ is embeddable with at most one crossing per edge if and only if the instance of 3-PARTITION has an affirmative answer.

Part "IF" of the claim is rather straightforward. We illustrate this with an instance of 3-PARTITION having 6 elements of weights $2,3,3,3,4,5$. This instance has a required 
partition $(3+3+4=10$ and $2+3+5=10)$ and the corresponding graph $G$ can be drawn with at most one crossing per edge as on Figure 3. In general, we draw graph $G$ as follows. First we draw transmitter and collector such that both are placed in the exterior face of each other. Then, we connect by thick edges in the exterior each third vertex of the transmitter to each $B$ th vertex of the collector, creating $m$ distinct faces. We assign the splitters to the faces according to the partition. Since the total size of each triple in the partition is $B$ and in each of the $m$ faces the collector has $B$ edges in the exterior circle, we can assign the edges of splitters to the sectors of the collector such that each edge will be crossed only once.

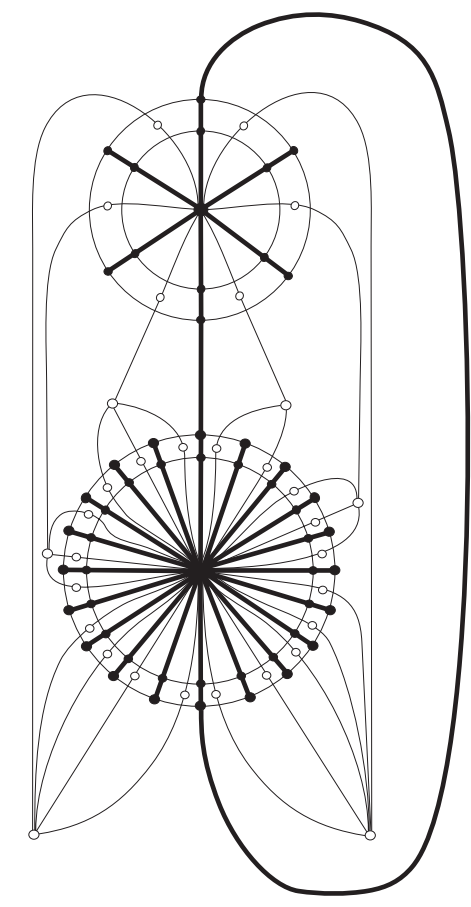

Figure 3: Instance with $A=\{2,3,3,3,4,5\}$.

Now we prove part "ONLY IF" of the statement. Consider a drawing of $G$ having at most one crossing per edge and let us construct the corresponding partition for 3PARTITION.

First, let us analyze the possible ways of drawing $G$. It is convenient to consider the possible drawings on a globe. It is well known that a sphere drawing has an equivalent planar representation with respect to the edge crossings; see [8, Proposition 8.3.1]. Without loss of generality we can assume that the transmitter center is a North Pole and the collector center is a South Pole of the globe. By construction, the globe is partitioned by the thick non-crossable meridian paths into $m$ distinct faces $F_{1}, F_{2}, \ldots F_{m}$. Moreover, since these meridian paths are non-crossable, the ordering of the meridian paths on the globe and the ordering of the faces on the globe correspond to the vertex ordering in the exterior circuits of the transmitter and collector. 
Now, let us find out how can we draw the thick paths of the transmitter adjacent to the North Pole but not participating in the meridian paths. Consider four consequent vertices of the exterior circuit of the transmitter, for instance, $t_{3 m}, t_{1}, t_{2}, t_{3}$. By construction and observation above, $t_{3 m}$ and $t_{3}$ are the vertices on two consequent meridian paths. These two meridian paths form one of the distinct faces, say $F_{1}$. Vertices $t_{1}$ and $t_{2}$ in the drawing must be placed in $F_{1}$ otherwise at least one of the meridian paths will be crossed. Moreover, since the transmitter is a "double"-wheel, the ordering of the thick paths adjacent to the North Pole and ending in $t_{1}$ and $t_{2}$ must be consistent with the ordering of the vertices in the exterior circuit of the transmitter. The same arguments work for all other consequent four-tuples of the exterior circuit of transmitter. This implies that there is a unique way of drawing the transmitter around the North Pole. Similarly, there is a unique way of drawing the collector around the South Pole. We also notice, that since we can not cross thick edges and other edges can be crossed at most once, we do not have any intersections between transmitter and collector.

Now, consider a drawing of the splitters. In any face $F_{i}, i \in\{1,2, \ldots, m\}$, we can place at most 3 splitters, otherwise one of the edges of the transmitter will be crossed more than once. The center of a splitter must be placed in the exterior of the transmitter and collector, otherwise one of the splitter edges will be crossed more than once. Hence, for each face $F_{i}, i \in\{1,2, \ldots, m\}$, the number of paths between the South Pole and the centers of three splitter assigned to $F_{i}$ is at most $B$. Since we have in total $B m$ such paths, each face contains exactly three splitters with exactly $B$ paths between the splitter centers and the South Pole.

Consider a partition of set $A$ correspondent to the assignment of splitters to the faces. By observation above, each triple of numbers correspondent to three splitters assigned to a face sums to $B$. Therefore, $A$ has a required partition. It remains to notice that 3PARTITION is strongly NP-complete and we are allowed to use unary encoding to describe the inputs of the problems. Hence, the reduction was polynomial.

Corollary 4.1.1. When $P \neq N P$, there does not exist a polynomial time 2-approximation algorithm for finding the crossing parameter of a graph on the plane.

Notice, however, that several natural classes of graphs have a bounded crossing parameter on the plane. For instance, graphs of intersections of objects in the plane with bounded objects density (disk graphs with bounded density are special case of these); graphs with bounded degree and bounded tree width; planar graphs.

Observation 4.1. The class of graphs with an embedding on the plane with crossing parameter 1 is not closed under taking minors. In fact, every graph is a minor of a graph with crossing parameter 1: take any good embedding, and then add a new vertex of degree two between every two successive crossings.

From work on the crossing number of graphs (the minimum total number of crossings in a planar embedding), we can also obtain bounds on the crossing parameter (on the 
plane). E.g., the crossing number of a complete graph with $n$ vertices is $\Theta\left(n^{4}\right)[9]$, hence its crossing parameter is $\Theta\left(n^{2}\right)$.

\section{Conclusions and Open Problems}

For several classes of graphs, it is now known that there are polynomial time approximation schemes for a large collection of problems. Each of these build upon the work by Baker [1]. In this paper, we gave a new class of graphs where the same approach can be used. An interesting question is whether there is an general notion under which the different results of the type can be unified.

A disadvantage of our algorithm is that an embedding with bounded crossings per edge is requested as part of the input. As discussed earlier, for some applications, we indeed get such an embedding. However, it would be interesting if "robust" versions of the algorithms can be designed, i.e., algorithms that do not need the embedding as part of the input. Note that such a robust PTAS has been designed by Nieberg et al. for the dominating set problem on unit disk graphs [10].

Recent work (see e.g., [2]) shows that there is a PTAS for the connected dominating set problem and other related problems on planar graphs and generalizations of it. It would be interesting to see if these results carry over to graphs with bounded crossing parameter.

\section{References}

[1] B. Baker. Approximation algorithms for NP-complete problems on planar graphs. Journal of the ACM 41 (1994), 153-180. Preliminary version in FOCS'83.

[2] E. Demaine and M. Hajiaghayi, Bidimensionality: New Connections between FPT Algorithms and PTASs. In Proceedings of the 16th Annual ACM-SIAM Symposium on Discrete Algorithms (SODA 2005), Vancouver, British Columbia, Canada, January 23-25, 2005, to appear.

[3] E. Demaine and M. Hajiaghayi, Graphs Excluding a Fixed Minor have Grids as Large as Treewidth, with Combinatorial and Algorithmic Applications through Bidimensionality. In Proceedings of the 16th Annual ACM-SIAM Symposium on Discrete Algorithms (SODA 2005), Vancouver, British Columbia, Canada, January 23-25, 2005, to appear.

[4] E. Demaine, N. Nishimura, M. Hajiaghayi, P. Ragde and D.M. Thilikos, Approximation algorithms for classes of graphs excluding single-crossing graphs as minors. To appear in J. Comp. System Sc.

[5] M.R. Garey, D.S. Johnson. Computers and intractability: A guide to the theory of NP-completeness. W.H. Freeman, San Francisco (1979). 
[6] M. Grohe. Local tree-width, excluded minors, and approximation algorithms. Combinatorica 23 (2003), 613-632.

[7] D. Eppstein. Diameter and treewidth in minor-closed graph families. Algorithmica 27 (2000) 275-291.

[8] J. Gross and J. Yellen. Graph Theory and Its Applications. New York, CRC Press (1999).

[9] F.T. Leighton. New lower bound techniques for VLSI. Mathematical Systems Theory 17 (1984) 47-70.

[10] T. Nieberg, J.L. Hurink, W. Kern. A robust PTAS for Maximum Independent Sets in unit disk graphs. In Proceedings of the 30th Workshop on Graph Theoretic Concepts in Computer Science, Bad Honnef, WG2004, Germany, Springer LNCS. To appear.

[11] J. Pach, G. Toth. Graphs drawn with few crossings per edge. Combinatorica 17 (1997) 427-439.

[12] J. A. Telle and A. Proskurowski. Algorithms for vertex partitioning problems on partial $k$-trees. SIAM J. Disc. Math. 17 (1997) 529-550.

[13] World Wide Web: http://mathworld.wolfram.com/GraphCrossing Number.html 


\section{Appendix}

Theorem 6.1. In any planar embedding of $K_{6}$ having at most one crossing per edge, between any two vertices there exists a path such that all edges in that path are crossed.

Proof. First we prove that each vertex is contained in at least two distinct crossed edges. Assume this is not true and there is an embedding of $K_{6}$ such that for some vertex $v_{1}$ edges $e_{1}=\left(v_{1}, v_{2}\right), e_{2}=\left(v_{1}, v_{3}\right), e_{3}=\left(v_{1}, v_{4}\right), e_{4}=\left(v_{1}, v_{5}\right)$ are not crossed by any edge. Without loss of generality, we assume that edges $e_{1}, e_{2}, e_{3}, e_{4}$ are drawn clockwise in this particular order. Since graph is complete, there is a simple cycle formed by edges $e_{1}, e_{3}$ and $e_{5}=\left(v_{2}, v_{4}\right)$ with only one edge $e_{5}$ that can be crossed. Vertices $v_{3}$ and $v_{5}$ belong to the different faces formed by that cycle. Therefore edge $\left(v_{3}, v_{5}\right)$ crosses $e_{5}$. On the other hand, there is a vertex $v_{6}$ that must be connected to both $v_{3}$ and $v_{5}$. Hence $e_{5}$ is crossed at least twice that leads to the contradiction.

Now, we prove that crossed edges form a connected graph. For a contradiction we assume that there are 2 or more connectivity components. Since every vertex is contained in two distinct crossed edges, each connectivity component has at least 3 vertices. Therefore, we can have only two components with 3 vertices each. Moreover, each component forms a triangle (cycle). Hence, the question is whether we can cross two triangles with curved sides such that each side of each triangle will be crossed exactly once? Take a side $\left(v_{1}, v_{2}\right)$ of triangle 1 . Vertices $v_{1}$ and $v_{2}$ belong to different faces formed by triangle 2 . The third vertex $v_{3}$ of triangle 1 will share the face either with $v_{1}$ or with $v_{2}$. Therefore, either edge $\left(v_{3}, v_{1}\right)$ or edge $\left(v_{3}, v_{2}\right)$ will cross the boundary of triangle 2 even number of times which contradicts to the requirement that each edge is crossed once. Therefore, crossed edges in $K_{6}$ form a connected graph as required.

The reader may even verify that, when $K_{6}$ is drawn with at most one crossing per edge, the crossing edges form a Hamiltonian circuit. This observation is out of the scope of this article and we leave it without a proof. 